\title{
Developing Codes to Capture Post-Operative Care
}

Ateev Mehrotra, Courtney A. Gidengil, Lee H. Hilborne, Ashley M. Kranz, Stephanie Stratos, John N. Mafi, Barbara O. Wynn 


\section{Library of Congress Cataloging-in-Publication Data is available for this publication.}

ISBN: 978-0-8330-9541-1

Published by the RAND Corporation, Santa Monica, Calif.

(C) Copyright 2016 RAND Corporation

RAND $^{\circledR}$ is a registered trademark.

\section{Limited Print and Electronic Distribution Rights}

This document and trademark(s) contained herein are protected by law. This representation of RAND intellectual property is provided for noncommercial use only. Unauthorized posting of this publication online is prohibited. Permission is given to duplicate this document for personal use only, as long as it is unaltered and complete. Permission is required from RAND to reproduce, or reuse in another form, any of its research documents for commercial use. For information on reprint and linking permissions, please visit www.rand.org/pubs/permissions.

The RAND Corporation is a research organization that develops solutions to public policy challenges to help make communities throughout the world safer and more secure, healthier and more prosperous. RAND is nonprofit, nonpartisan, and committed to the public interest.

RAND's publications do not necessarily reflect the opinions of its research clients and sponsors.

Support RAND

Make a tax-deductible charitable contribution at www.rand.org/giving/contribute

www.rand.org 
The Centers for Medicare \& Medicaid Services (CMS) uses the resource-based relative value system (RBRVS) to determine payment for physicians and nonphysician practitioners for their professional services. The relative values for physician work measure the relative levels of professional time, effort, skill, and stress associated with providing services. For many surgeries and other types of procedures, Medicare payment also covers a bundle of post-operative visits delivered during a global period of 10 days or 90 days anchored on the surgery date. In the final rule for the 2015 physician fee schedule, CMS announced that all surgeries with a 10- or 90-day global period would transition to a 0-day global period in 2017 and 2018, respectively. Under this policy, physicians would bill separately for any post-operative visits after the day of surgery. CMS's rationale for scaling back global surgical packages was driven by concerns over the accuracy of the payment for post-operative care. In Section 523 of the Medicare Access and CHIP Reauthorization Act of 2015 (MACRA), Congress directed CMS not to transition all 10day and 90-day global surgery packages to 0-day global periods (Public Law 114-10, 2015). Instead, Congress mandated that CMS develop and implement a process to gather the necessary data to appropriately value post-operative care. Congress has required CMS to collect data on the "number and level" of visits in the global period from a representative sample of physicians beginning January 1, 2017. Congress suggested, but does not require, that the data be collected through claims.

CMS asked the RAND Corporation to provide recommendations on how to best collect the number and level of post-operative visits through the use of nonpayment claims (recommendations on alternatives to nonpayment claims for data collection are outside the scope of this report). To do so, RAND gathered input from individual proceduralists and experts to describe the range of post-operative care provided during the global period. In this report, we detail this background research and RAND's recommendations for a set of eight nonpayment codes for post-operative care.

The findings should be of interest to health policymakers, representatives of physician and nonphysician practitioner professional associations, and health services researchers.

This study was funded by CMS under contract HHSM-500-2012-00163G with Chava Sheffield as project officer. This research was conducted in RAND Health, a division of the RAND Corporation. A profile of RAND Health, abstracts of its publications, and ordering information can be found at www.rand.org/health. 


\section{Table of Contents}

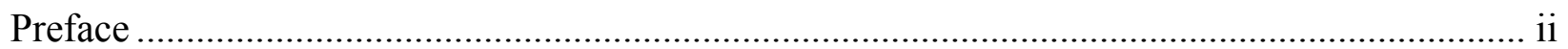

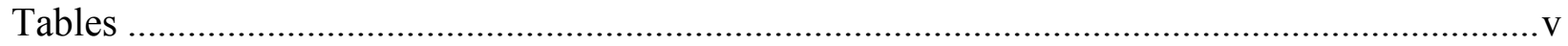

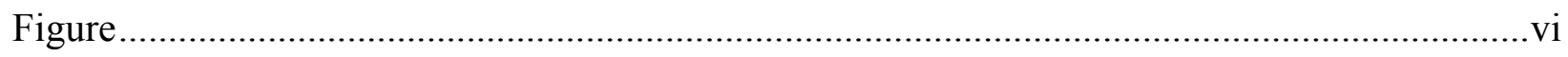

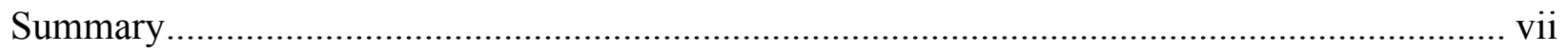

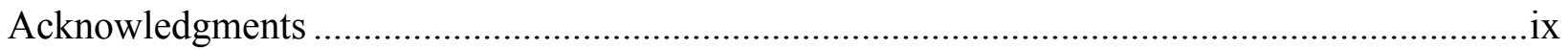

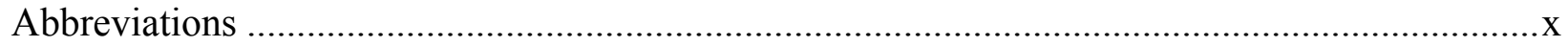

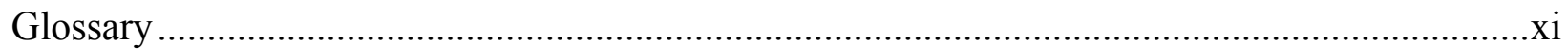

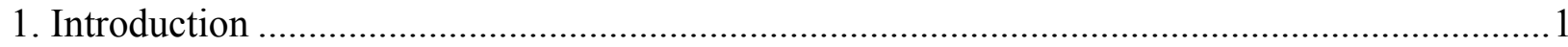

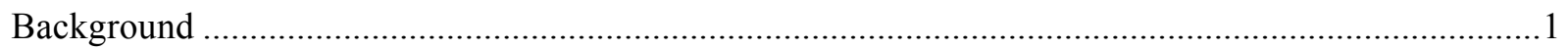

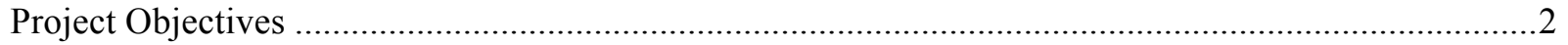

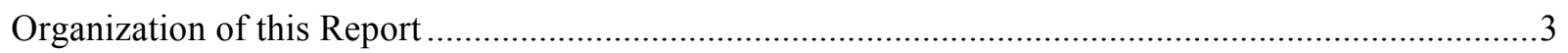

Clarifications on Terminology ...............................................................................................

2. Overview of Current Payment Policy for Post-Operative Services in the Global Period ...........5

Process for Determining Valuation of Global Procedures ....................................................................5

Billing for Post-Operative Services Provided During the Global Period................................................7

3. Interviews on Post-Operative Care ................................................................................. 10

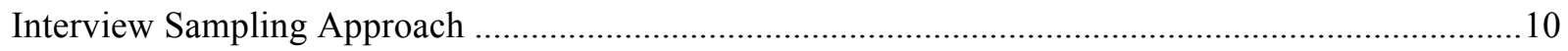

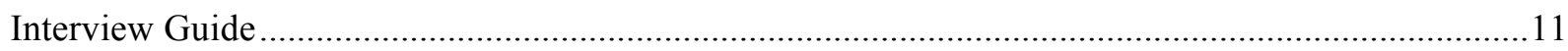

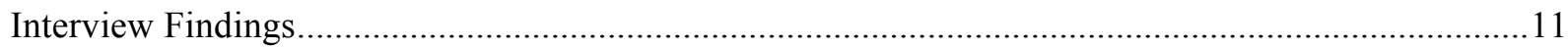

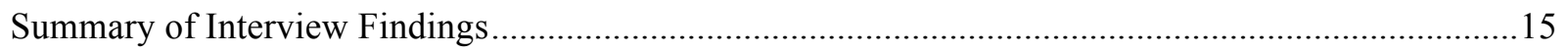

4. Challenges Identified During Interviews and Expert Panel in Capturing Post-Operative

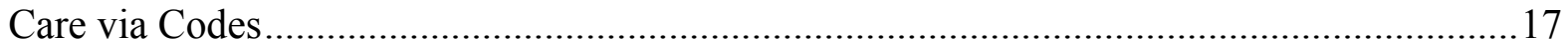

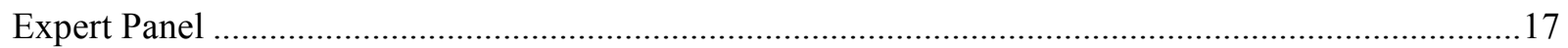

Challenges Identified During the Interviews and/or Expert Panel...................................................18

Summary of Challenges Associated with Capturing Post-Operative Care ..........................................23

5. Options for Capturing Post-Operative Care .......................................................................24

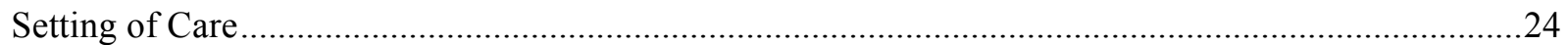

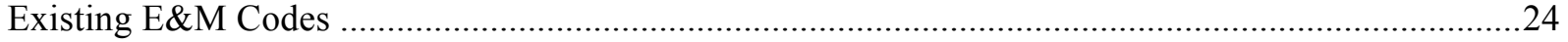

Scope of Care

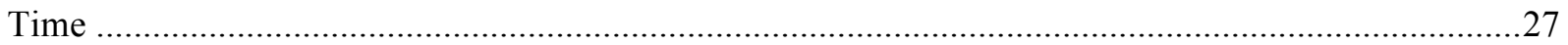

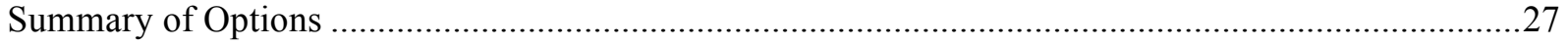

6. Recommendations for Capturing Post-Operative Care ....................................................28

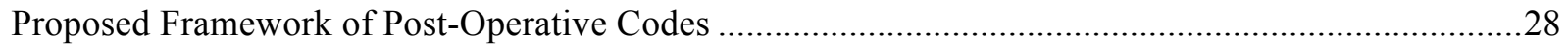

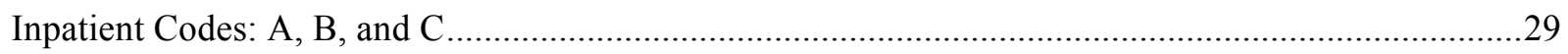

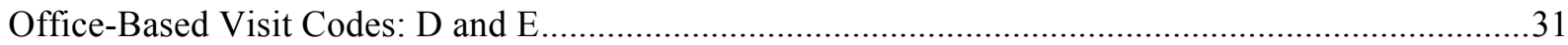

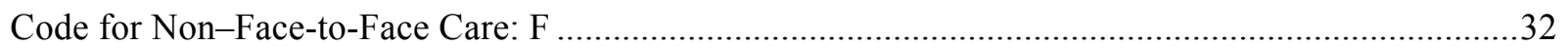

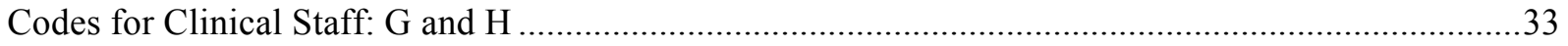




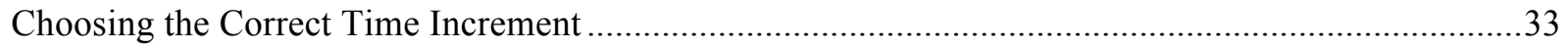

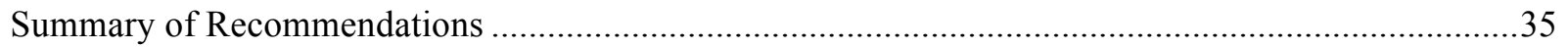

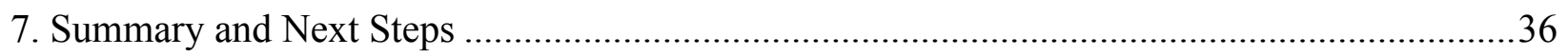

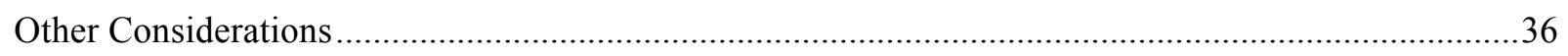

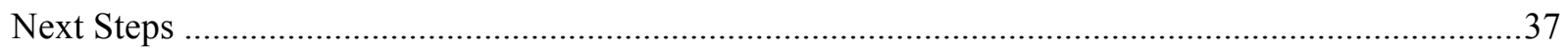

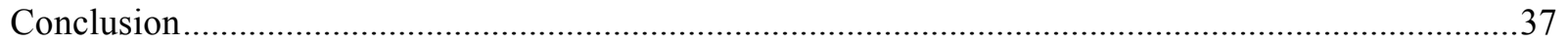

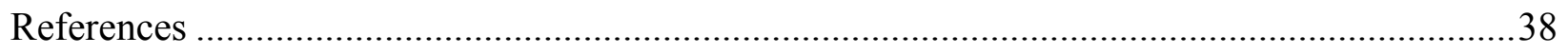




\section{Tables}

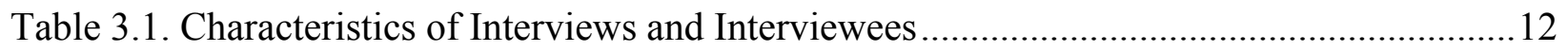

Table 4.1. 2015 Global Surgical E\&M Utilization Percentage by CPT Code ............................21

Table 6.1. Visit Length Over Time from 1997 to 2012 for Medicare Patients ............................34 
Figure

Figure 6.1. Proposed Post-Operative Visit Codes .................................................................29 


\section{Summary}

The Centers for Medicare \& Medicaid Services (CMS) uses the resource-based relative value system (RBRVS) to determine payment for physicians and nonphysician practitioners (NPPs) for their professional services. The relative values for physician work measure the relative levels of professional time, effort, skill and stress, associated with providing services. For many surgeries and other types of procedures, Medicare payment also covers a bundle of post-operative visits delivered during a global period of 10 days or 90 days anchored on a surgery date. In the final rule for the 2015 physician fee schedule, CMS announced that all surgeries with a 10- or 90-day global period would transition to a 0-day global period in 2017 and 2018, respectively. Under this policy, physicians would bill separately for any post-operative visits after the day of surgery. CMS's rationale for scaling back global surgical packages was driven by concerns over the accuracy of the payment for post-operative care. In Section 523 of the Medicare Access and CHIP Reauthorization Act of 2015 (MACRA), Congress directed CMS not to transition all 10day and 90-day global surgery packages to 0-day global periods (Public Law 114-10, 2015). Instead, Congress mandated that CMS develop and implement a process to gather the necessary data to appropriately value post-operative care. Congress has required CMS to collect data on the "number and level" of visits in the global period from a representative sample of physicians beginning January 1, 2017. Congress suggested, but does not require, that the data be collected through claims.

CMS asked the RAND Corporation to provide recommendations on how to best collect the number and level of post-operative visits through the use of nonpayment claims (recommendations on alternatives to nonpayment claims for data collection are outside the scope of this report). To do so, RAND first gathered input from individual proceduralists and later an expert panel to describe the range of post-operative care provided during the global period.

Interviewees described differences in post-operative care by type of procedure, care setting, geography, and patient, provider, and practice characteristics. Despite these differences, respondents did report some similarity in post-operative care delivered. For example, postoperative care included a set of similar tasks (e.g., taking history, reviewing vitals, checking wounds, managing pain). Most respondents also agreed that post-operative care in inpatient settings was more work than post-operative care in office-based settings.

Both the interviews and the expert panel identified multiple challenges in capturing postoperative care, including: (1) who performs the work provided (proceduralist versus non proceduralist physician versus NPP versus clinical staff), as well as the heterogeneity of the work itself; (2) how to capture practice expenses, such as staff time, space, equipment, and supplies; (3) how to capture the variability in the complexity of post-operative care; and (4) the need for a reporting system with a burden low enough so as not to discourage participation. 
Based on input from the interviews and the expert panel, we propose a new set of codes that combine scope of services with time, for both inpatient and office-based services. In the inpatient setting, visits are divided into typical, complex, and critical care encounters. In the office-based setting, visits are divided into typical and complex encounters, as well visits during which patients are seen only by clinical staff. In both settings, typical visits would be used most frequently and we have provided a list of services that would be encompassed by a typical visit. We also have allowed for the ability to code Internet/phone-based care delivery with additional codes. The recommended codes attempt to balance the need for a simple and straightforward system with the demand for a set of codes to capture the granularity and heterogeneity associated with post-operative care delivery. Moving forward, it will be useful to test these codes with proceduralists to ensure that they are feasible, reliable, and capture the full range of postoperative care. 


\section{Acknowledgments}

We thank our interviewees and expert panel for sharing their time, valuable insights, and critical feedback. We would like to thank the following specialty societies for assisting with recruitment of interviewees: the American Academy of Dermatology, the American Academy of Family Physicians, the American Academy of Nurse Practitioners, the American Academy of Ophthalmology, the American Academy of Orthopaedic Surgeons, the American Academy of Otolaryngology, the American Academy of Physician Assistants, the American Association of Neurological Surgeons, the American College of Cardiology, the American College of Surgeons, the American Medical Association, the American Podiatric Medical Association, the American Society of Anesthesiologists, the American Society of Plastic Surgeons, the American Urological Association, the Society of Hospital Medicine, the Society of Thoracic Surgeons, the Society for Vascular Surgery, and the Wound, Ostomy and Continence Nurses Society.

Our acknowledgment of their contributions does not imply that these individuals or organizations endorse the contents of this report. 


\section{Abbreviations}

AMA

CMS

$\mathrm{CPT}^{\circledR}$

EMR

E\&M

ICU

MACRA

NAMCS

NP

NPP

OR

PA

PE

RBRVS

$\mathrm{RN}$

RUC

RVU
American Medical Association

Centers for Medicare \& Medicaid Services

Current Procedural Terminology

electronic medical record

evaluation and management

intensive care unit

Medicare Access and CHIP Reauthorization Act

National Ambulatory Medical Care Survey

nurse practitioner

nonphysician practitioner

operating room

physician assistant

practice expense

resource-based relative value scale

registered nurse

[American Medical Association/Specialty Society] Relative [Value Scale] Update Committee

relative value unit 


\section{Glossary}

\section{Word}

Clinical staff

Code/procedure/services

Global period

Intensity

Nonphysician practitioner

Physician

Post-operative work

\section{Definition}

Defined as registered nurses, licensed practical nurses, medical technical assistants, and other health professionals who are not separately payable but whose services may be covered "incident to" a physician or nonphysician practitioner's service.

These terms are generally interchangeable in this report. A service is described by a procedure code (Current Procedural Terminology ${ }^{\circledR}$ or Healthcare Common Procedure Coding System).

Surgical procedures may have a 10- or 90-day global period during which follow-up post-operative visits are bundled into the payment for the procedure. A 90-day global period also includes services provided on the day before the procedure. Some surgical procedures have a 0day period, meaning that physicians/nonphysician practitioners would bill separately for any post-operative visits after the day of surgery.

Refers to the cognitive effort and judgment, technical skill and physical effort, and stress due to potential patient risk associated with delivering a service.

Refers to practitioners other than physicians, such as physician assistants, nurse practitioners, certified nurse midwives, and clinical nurse specialists, who can bill Medicare for services performed consistent with their state scope of practice and in certain circumstances.

Defined as doctors of medicine, doctors of osteopathy, doctors of dental surgery or of dental medicine, doctors of podiatric medicine, and doctors of optometry.

Work related to the surgical procedure after a patient's discharge from the recovery room through the end of the global period. 
Proceduralist

Resource-based relative value system (RBRVS)

Teaching physician

Work
Physicians and nonphysician practitioners who perform procedures associated with a global period, including surgeons, podiatrists, cardiologists, and gastroenterologists. We use the term proceduralist instead of surgeons because nonsurgeons, such as cardiologists and gastroenterologists commonly perform procedures associated with a global period.

A system for determining payments to physicians for treating Medicare patients that takes into account the work done by the physicians, malpractice insurance, and practice expenses including staff salaries, overhead, supplies, and equipment.

A physician (other than another resident) who involves residents in the care of his or her patients.

Defined as the physician's or nonphysician practitioner's time, effort, skills, and stress associated with providing the service. 


\section{Introduction}

\section{Background}

Most physician services are defined using the Current Procedural Terminology $\left(\mathrm{CPT}^{\circledR}\right)$ codes $^{\circledR}$ from the American Medical Association (AMA). Medicare's physician payment for surgical and other procedures covers a physician's work involved in delivering the service, practice expenses (PEs), and malpractice expenses. The work component includes the surgery, preparations prior to the surgery, and immediate post-operative recovery. For many surgeries and other types of procedures, Medicare also covers a bundle of post-operative visits delivered during a global period of 10 days or 90 days anchored on the surgery date. Other physicians (e.g., hospitalists, intensivists, pain specialists, primary care physicians) and nonphysician practitioners (NPPs) providing post-operative care for the same patient during the 90 days are paid separately. Payment for the care of complications arising from the surgery is included in the 90-day global payment unless the complications require another visit to the operating room (OR). All of the components of the surgical care, including the surgery and post-operative care, are measured in relative value units (RVUs) and then converted to a dollar payment rate using a constant conversion factor.

For example, the Medicare payment for total knee arthroplasty (CPT code 27447) was in part determined by the assumption that seven post-operative visits (three inpatient, one hospital discharge, three office-based) typically occur within 90 days following the surgery. Physicians who perform the surgery cannot bill for post-operative visits, thus, they receive the same fixed payment for a knee arthroplasty regardless of how many post-operative visits actually take place within the 90-day global period.

Information on the post-operative visits provided by the performing proceduralist during the global period is not required to be reported, therefore, it cannot be tracked in Medicare claims data. In two studies of medical charts, the Office of the Inspector General found that the number of post-operative visits actually provided by proceduralists to patients fell short of the number of visits included in the global surgical package for cardiovascular and musculoskeletal procedures (Office of the Inspector General, 2012a and 2012b). The goal of a bundled payment system is to encourage efficiency, so the fewer number of visits observed by the Office of the Inspector General could be reflective of these efficiency gains. However, the concern is that the bundled payment has not been reduced to reflect these efficiency gains.

Moreover, as the health care delivery system evolves into more team-based care, there has been an increase in billings by hospitalists, intensivists, and NPPs (Cromwell et al., 2010). For example, the number of visits by intensivists to patients in the post-operative period has increased by 30 percent to more than 100 percent across many surgeries. This increase has 
occurred despite the fact that the age, gender, and racial mix of these patients have not changed over time (Cromwell et al., 2010). The services provided by these physicians/NPPs may be substituting for the post-operative care typically provided by the proceduralist. Together with the findings of the Office of the Inspector General, these changes raise concern that Centers for Medicare \& Medicaid Services (CMS) may be paying twice for some post-operative care, once to the proceduralist who performed the surgery (as part of the global payment) and again to these other physicians/NPPs.

In the final rule for the 2015 physician fee schedule, CMS announced that all surgeries with a 10- or 90-day global period would transition to a 0-day global period in 2017 and 2018, respectively. Under this policy, physicians would bill separately for any post-operative visits that occur after the day of surgery. CMS's rationale for scaling back global surgical packages was driven by concerns over the accuracy of the valuations for post-operative care and the global surgical packages. Moreover, the structure of the global packages does not reflect the changes in how post-operative care is provided or changes in the health care delivery system. With the shift of care to larger group practices and team-based care environments as noted earlier, the models of post-operative care have become increasingly heterogeneous, raising ongoing challenges in defining, valuing, and updating the "typical" number and level of post-operative visits that should be included in the global package (Code of Federal Regulations, 2014).

In Section 523 of the Medicare Access and CHIP Reauthorization Act of 2015 (MACRA), Congress directed CMS not to transition all 10-day and 90-day global surgery packages to 0-day global periods (Public Law 114-10, 2015). Instead, Congress mandated that CMS develop and implement a process to gather the necessary data to appropriately value surgical care. There are several key components of the law related to this data collection:

- Data collection should begin no later than January 1, 2017.

- Data must be collected from a representative sample of physicians.

- There should be collection on both the number and level of medical visits furnished during the global period.

- Congress suggested this should be reported on claims, but provided flexibility to CMS to determine the collection methodology.

- The Inspector General will audit a sample of the information reported.

- For 2019 payments and beyond, CMS will use the information reported by this data collection to improve the accuracy of valuation of surgical services.

\section{Project Objectives}

CMS asked the RAND Corporation to provide recommendations on how best to collect data on post-operative care through reporting on claims. CPT code 99024 is a Medicare nonpayment code that can be used to report post-operative visits. However, as currently defined, it indicates only that a visit was provided but not the level of the visit. RAND's objective was to propose a set of descriptive categories to capture the major differences in the breadth and intensity of post- 
operative care provided during the global periods. To create these categories, RAND first gathered input from individual proceduralists and later an expert panel to describe the range of post-operative care provided during the global period. These categories could be translated into nonpayment procedure codes that would be submitted by proceduralists who provide postoperative care during the global period to CMS. It is important to emphasize that the purpose of these codes is for data collection and not for payment.

Issues regarding alternatives to nonpayment codes for data collection, the implementation of these codes in 2017, and how the information will be used to improve the valuation of surgical services starting in 2019 are beyond the scope of the current work. Other issues outside the scope of this report include: (1) who will submit the nonpayment procedure codes; (2) for which procedures reported information will be collected; and (3) how the Inspector General will judge the accuracy of the reported information submitted.

\section{Organization of this Report}

The report is organized as follows:

- Chapter 1 summarizes the background for this work and the objectives of this project.

- Chapter 2 provides an overview of Medicare payment policy for procedures associated with a global period, as well as how those payments are currently determined.

- Chapter 3 summarizes our findings from interviews with proceduralists who receive global surgery payments, as well as other physicians/NPPs who provide post-operative care.

- Chapter 4 summarizes key challenges identified during both interviews and a subsequent expert panel in capturing post-operative care via claims.

- Chapter 5 lays out four options for capturing post-operative care, along with their strengths and weaknesses.

- Chapter 6 provides RAND's recommendations on capturing post-operative care using claims.

- Chapter 7 summarizes our findings and details key next steps.

\section{Clarifications on Terminology}

Procedures associated with a global period are performed by a variety of physicians including surgeons, podiatrists, cardiologists, gastroenterologists, as well as NPPs. In an effort to distinguish between those who perform procedures and those who provide post-operative care but do not perform procedures, we use the term proceduralists in this report. We use the term proceduralist instead of surgeon because nonsurgeons, such as cardiologists, commonly perform procedures associated with a global period.

We define those who can bill under the physician fee schedule as physicians/NPPs and those who provide clinical service but cannot bill Medicare as clinical staff. For purposes of this report, the term "physician" is limited to doctors of medicine, doctors of osteopathy, doctors of 
dental surgery or of dental medicine, doctors of podiatric medicine, and doctors of optometry. NPPs include practitioners other than physicians, such as physician assistants (PAs), nurse practitioners (NPs), certified nurse midwives, and clinical nurse specialists, who can bill Medicare for services performed consistent with their state scope of practice and in certain circumstances. Clinical staff include registered nurses (RNs), licensed practical nurses, medical technical assistants, and other health professionals whose services are not separately payable but whose services may be billed for as "incident to" a physician/NPP's service. These include clinical staff employed by the physician/NPP.

We use the term post-operative care to capture all care provided after the immediate postoperative period (e.g., recovery room) (CMS, 2015; Medicare Learning Network, 2015). This care can be provided in follow-up visits in a hospital or in an office, including in the outpatient hospital setting. 


\section{Overview of Current Payment Policy for Post-Operative Services in the Global Period}

CMS uses the resource-based relative value system (RBRVS) to determine reimbursement for physicians/NPPs for their professional services. Under RBRVS, payment for a specific service is broken into three elements: physician work, PE, and malpractice expense. Each component is valued in RVUs. Total RVUs are adjusted for geographic price differences and multiplied by a dollar conversion factor to determine the Medicare physician fee schedule amount. In the case of surgeries and other procedures, the physician work component includes the procedure itself, preparations prior to the procedure, and immediate post-operative recovery.

The work RVUs measure the relative levels of physician time, effort, skill, and stress associated with providing the service. The PE RVUs measure the relative costs of resource inputs used in providing a service, including office rent, nonphysician personnel labor, equipment, and supplies.

Unlike other services, surgical procedures may include post-operative visits within a global period (10 or 90 days following the surgical procedure) and, in the case of surgeries with 90-day global periods, a pre-operative visit the day before the procedure. Post-operative evaluation and management (E\&M) visits related to surgical procedures are bundled into total work for CPT procedure codes with a 10- or 90-day global period. These E\&M visits are not paid separately under RBRVS when they are performed by the same proceduralist who performed the surgical procedure. The next section describes in further detail the process for determining the value of global procedures.

\section{Process for Determining Valuation of Global Procedures}

After the RBRVS was implemented, a process to review and revise the RVUs was needed because continuing refinement and updating are required as CPT codes are modified or added, new technology is introduced, and practice patterns change. The [American Medical Association/Specialty Society] Relative [Value Scale] Update Committee (RUC) meets three times a year to consider new and revised CPT codes and potentially misvalued services that were identified either through its Relativity Assessment Workgroup or by CMS. The RUC is supported by an Advisory Committee of 123 specialty societies that collect data and make recommendations on the work and PE RVUs for the codes that the RUC has referred to them. The RUC reviews the specialty society recommendations and makes recommendations to CMS (AMA, 2016). 


\section{Estimating Physician/NPP Work}

The predominant mechanism to establish new or revised RVUs is via physician surveys conducted by the specialty societies using a vignette that describes the typical patient receiving the procedure. For post-operative visits, the survey elicits information on where the proceduralist typically provides the procedure (and in the case of a hospital procedure, whether the patient is discharged the same day or typically has an overnight stay), post-recovery room visits provided on the same day as the procedure, and the estimated number of post-operative visits performed in both the hospital and office-settings following the day of surgery.

The survey uses CPT codes for E\&M visits to collect the visit information. Typical times associated with each code for hospital visits (noncritical care inpatient visits, subsequent observation care visits, discharge day management) and office/clinic visits are provided in the survey. The respondent is also asked to select a procedure code that is most similar to the survey code. Notably, the respondent is asked to compare the survey procedure and reference procedure on different domains of intensity (mental effort and judgment, technical skill/physical effort, and psychological stress) using a scale of 1 to 5 for pre-service, intra-service, and immediate postoperative services; a similar ranking is not requested for the post-operative visits. The final survey question asks the respondent to estimate total work RVUs for the survey code using magnitude estimation and the work value for the reference code. Separate estimates of work for the individual components are not obtained.

The RUC uses a variety of methodologies to review the specialty-society proposals and formulate its recommendations for work values, including a comparison of the proposed values with values for key reference codes furnished by the specialty and other physician specialties. After reviewing the RUC's recommendations, CMS determines a final single work value for the surgical procedure that accounts for all components of the service, including the post-operative visits. Work for the post-operative visits is not separately estimated in the valuation process nor is there any assessment of how the post-operative visit work compares with the E\&M visits defined by the CPT codes used to estimate visits.

\section{Estimating PE}

In contrast, estimates of post-operative visits directly affect the PE values. For new, revised, or misvalued codes, the RUC PE subcommittee reviews estimates put forth by the specialty societies of the direct PE inputs for clinical staff, medical equipment, and supplies associated with each post-operative office visit for a given procedure. For example, the equipment estimate might include a cast cutter and the supplies estimate might include bandages and dressings. CMS reviews the RUC recommendations, develops refined direct cost inputs, and attaches prices to each input (e.g., by attaching current hourly rates to the estimated time for a nurse). For a surgical procedure performed in an office setting, a similar step is taken with regard to the direct 
PE inputs for the intraservice time. No direct costs are associated with intra-service time for procedures performed in a facility setting or hospital inpatient post-operative visits because the facility assumes those costs. Indirect costs are allocated to a surgical procedure on the basis of the direct costs specifically associated with a code and the work RVUs.

In theory, the PE direct cost estimates for a surgical procedure performed in an inpatient setting should therefore only reflect the number of post-operative office visits and any specialized staff, equipment, or supplies used during these visits (note that the 90-day global includes the day before the procedure, so it might include direct costs for this day of preoperative services). However, many estimates were made many years ago, and thus may not reflect current practices. The shift of surgical procedures from inpatient to the outpatient setting, along with shorter lengths of inpatient stay, may affect the number of post-operative office visits and type of care provided during such visits. In addition, the growth of team-based care has implications for the respective roles of physicians, NPPs, and clinical staff in providing postoperative care.

\section{Billing for Post-Operative Services Provided During the Global Period}

Medicare bundles post-operative care related to surgical procedures into the total payment for procedure codes with a 10- or 90-day global period. Post-operative visits are not paid separately under RBRVS when they are performed by the same proceduralist who performed the surgical procedure. ${ }^{1}$ The bundling policy applies to all medical and surgical services during the global period provided by the proceduralist who performed the surgical procedure, including follow-up visits, post-surgical pain management, miscellaneous services and supplies, and any additional medical and surgical services because of complications that do not require an additional trip to the OR. ${ }^{2}$

Currently, proceduralists do not need to bill for post-operative E\&M visits to receive payment for these services because they are included in the bundled payment for the surgical procedure. As a result, Medicare claims data do not indicate when post-operative E\&M visits are provided to patients.

The physician performing the surgery may be paid separately for noninpatient medically necessary E\&M visits during the global period if the service is documented as not related to the post-operative care of the surgery (modifier $=24$ ) or is documented as being more extensive than the usual pre-operative and post-operative care furnished on the same day as the procedure (modifier $=25)$.

\footnotetext{
${ }^{1}$ Proceduralists use modifier -54 to indicate that they performed the surgical procedure and inpatient E\&M visits, but not other E\&M visits. Physicians/NPPs use modifier -55 to indicate that they performed only post-operative outpatient E\&M services.

${ }^{2}$ For procedure codes with 0 -day global periods, any post-operative visits on the day of the surgery are bundled.
} 


\section{Billing When Multiple Physicians/NPPs Provide Post-Operative Care}

Medicare policies pertaining to situations where more than one physician/NPP provides postoperative care to a patient include:

- The Medicare fee schedule allocates the total RVUs for the global period between preoperative, surgical care only, and post-operative care. When the proceduralist transfers care to another physician/NPP who is not a member of the same group, payment for the surgical procedure is split between the proceduralist and the receiving physician/NPP. The proceduralist bills for surgical care only (modifier $=54$ ) while the physician/NPP assuming responsibility bills for the post-operative care beginning with the date that care is transferred (modifier $=55$ ). ${ }^{3}$ For example, if complex spinal surgery was performed in an urban hospital by an orthopedic surgeon on a rural beneficiary who was referred to a local physician in the rural community for post-discharge follow-up care, the orthopedic surgeon would bill using the -54 modifier and the local physician providing the followup care would bill using the -55 modifier. Another example would be an ophthalmologist who refers a beneficiary to an optometrist for post-operative services.

- Physicians in the same group practice who are in the same specialty bill are paid as though they are a single entity. In other words, services furnished by members of the group in the same specialty are considered post-operative services that are bundled into the global payment.

- Medically necessary services furnished by another physician/NPP during the global period are paid under the usual policies for services furnished (unless there is a transfer of care agreement). This includes services related to the surgery, visits unrelated to the diagnosis for which the surgery is performed, or treatment for the underlying condition that is not part of normal recovery from surgery.

Medicare billing rules do not explicitly address post-operative care provided by qualified NPPs who are in the same group practice or have the same employer. There are two different mechanisms for covering other services provided by NPPs. If NPPs acting within their state scopes of practice provide a medically necessary service that would be covered as a physician service if performed by an allopathic or osteopathic physician, the services are separately payable at 85 percent of the Medicare physician fee schedule amount. Alternatively, the services may meet the requirement for being "incident to" a physician's service, in which case they are

\footnotetext{
${ }^{3}$ The receiving physician/NPP cannot bill for any part of the global service until he or she has provided at least one service. Once the physician/NPP has seen the patient, the physician bills for post-operative services beginning with the date care was transferred. The contractor responsible for processing physician/NPP claims allocates the payment attributable to the post-operative services based on the date care was transferred. The sum of the payments cannot exceed the total payment for the surgical procedure. The proceduralist and the receiving physician are expected to coordinate their claims and retain documentation on the transfer of care. Where a transfer of care does not occur, CMS's contractor will determine whether the services of a receiving physician/NPP should be paid separately or denied for medical necessity reasons (CMS, 2015).
} 
included in the physician's bill and paid at 100 percent of the fee schedule amount. "Incident to" services are an integral part of a physician/NPP's professional services that are furnished by another individual (e.g., physician, NPP, or clinical staff) acting under their direct supervision. The physician/NPP must be present in the office suite and immediately available to provide assistance and direction. The services are included in the physician's bill. The billing policy allows for a shared E\&M visit if a physician and qualified NPPs within the same group practice or with the same employer each personally perform a substantive portion of an E\&M visit on the same date of service and the "incident to" requirements are met. If the physician is present in the office but does not see the patient during the visit, CPT code 99211 is billed for an "incident to" service. If the "incident to" requirements are not met, the service is only covered as an NPP service.

\section{Billing by Teaching Physicians}

In academic institutions, Medicare has policies regarding billings for direct patient care services provided by teaching physicians supervising residents in approved teaching programs. The general rule is that the teaching (also known as the attending) physician must be present for all key or critical portions of the service and remain immediately available for the duration of the care. Medicare has specific rules for supervising surgical procedures, but because there is no separate billing for post-operative services covered by the global payment, there are no rules for reporting these services. In contrast, when an E\&M service is provided and billed outside the global period, the teaching physician must be physically present during the portion of the service that determines the level of visit billed (history, physical examination, and medical decisionmaking). This raises two issues: (1) are residents providing post-operative services that substitute for services typically provided by a proceduralist in nonacademic settings; and, (2) what rules should apply to reporting post-operative visits furnished by residents acting under a teaching physician's supervision? 


\section{Interviews on Post-Operative Care}

To describe the range of post-operative care provided during the global period, we conducted a series of interviews with proceduralists and other physicians/NPPs involved in post-operative care. In this chapter, we describe our approach to sampling physicians/NPPs for the interviews, the interviewee characteristics, and the interview content. We also provide the interviewees' description of the post-operative care provided. Chapter 4 describes interviewees' input on the challenges of developing a set of codes to capture post-operative care.

\section{Interview Sampling Approach}

To identify the specialties that most commonly provide post-operative care within a global period, we calculated the total number of procedures performed within the fee-for-service Medicare program for each Healthcare Common Procedure Coding System code with a 10- or 90-day global period. We also used Medicare claims data to determine which specialties performed these procedures. The 12 specialties with the most work using global procedures were identified. In January and February 2016, we approached societies for these 12 specialties along with seven other organizations representing other physicians/NPPs involved in post-operative care and/or engaged in payment for post-operative care:

- Specialties that perform procedures:

- American Academy of Dermatology

- American Academy of Ophthalmology

- American Academy of Orthopaedic Surgeons

- American Academy of Otolaryngology

- American Association of Neurological Surgeons

- American College of Cardiology

- American College of Surgeons

- American Podiatric Medical Association

- American Society of Plastic Surgeons

- American Urological Association

- Society of Thoracic Surgeons

- Society for Vascular Surgery

- Other organizations

- American Academy of Family Physicians

- American Academy of Nurse Practitioners

- American Academy of Physician Assistants

- American Medical Association

- American Society of Anesthesiologists

- Society of Hospital Medicine

- Wound Ostomy and Continence Nurses Society 
Societies were asked to nominate members with and without payment policy expertise to participate in 30-minute semistructured interviews describing the range of post-operative care they provide for common procedures during the global period. We specifically requested nominations of proceduralists with and without payment policy expertise in order to obtain information from individuals both more and less familiar with the payment implications of the delivery of clinical care. We offered to conduct interviews via phone or in person at the CPT Editorial Panel meeting in February 2016 in Miami, Florida. This meeting was chosen because it coincided with our short timeline for interviews and had proceduralists representing a variety of specialists in attendance, not because we wanted to focus on those with CPT experience. No incentives for participation were provided.

\section{Interview Guide}

Interviewers utilized a semistructured interview guide. For interviewees who performed procedures, the interview was anchored on the most common surgical procedure with a global period performed by those in their specialty or a common procedure the interviewee performed that had a 10- or 90-day global period. Interviewees who did not perform procedures were asked to describe the post-operative care they provided for procedures with global periods. Additional procedures were discussed when time permitted. Interviewers were directed to prompt about

- activities and services provided beginning on the day of the procedure

- location of care provided

- length of visits

- care from other physicians/NPPs and clinical staff

- supplies and equipment used.

In addition, interviewees were asked for ideas on how to collect post-operative visit data through claims-based reporting. We also collected basic demographic information on practice type, location, and percentage of patients insured by Medicare. Identities of interviewees were kept confidential.

\section{Interview Findings}

Interviews were conducted with 33 proceduralists and other physicians/NPPs involved in post-operative care from January to March 2016. Characteristics of interviews and interviewees are described in Table 3.1. Interviewees practice in 22 states, 44 percent work in multispecialty groups, and 52 percent said that more than half of their patients have Medicare. Interview length ranged from 25 minutes to more than 90 minutes. A wide range of procedures was discussed during interviews including Whipple, total joint replacement, cataract surgery, robotic prostatectomy, lumbar fusion, coronary artery bypass graft surgery, carotid endarterectomy, and 
punch biopsy. Approximately 82 percent of interviews included discussion of a procedure with a 90-day global period. Interviewees reported delivering 0 to more than 15 post-operative office visits during the global period.

Table 3.1. Characteristics of Interviews and Interviewees ${ }^{a}$

\section{Characteristics}

Total interviews

Range of interview length (minutes)

$\%$ Interviews discussing a procedure with a 10-day global ${ }^{\mathrm{a}}$

$\%$ Interviews discussing a procedure with a 90-day global ${ }^{\mathrm{a}}$

$\%$ Interviewees who perform procedures

Number of office visits provided during post-operative period (range)

Region of practice

$\%$ Northeast

$\%$ Midwest

$\%$ South

$\%$ West

$\%$ Male

$\%$ With $\geq 50 \%$ Medicare patients

$\%$ In group with $<20$ employees

Practice type

$\%$ In multispecialty group
(N)

33

25 to $>90$

$24 \%(8)$

$82 \%(27)$

91\% (30)

0-15

$27 \%(9)$

$21 \%(7)$

$33 \%(11)$

$18 \%(6)$

$88 \%(29)$

$52 \%(17)$

$44 \%(14)$

$44 \%$ (14) 
$\%$ In single specialty group

$\%$ In hospital-based practice

$\%$ Affiliated with a teaching hospital
$36 \%(12)$

$22 \%(7)$

$52 \%(17)$

SOURCE: Authors' analysis.

${ }^{\text {a }}$ Some interviews included a discussion of multiple procedures. Percentages may also add up to greater than $100 \%$ due to rounding.

\section{Scope of Post-Operative Care Activities}

During the interviews, interviewees discussed a broad range of post-operative care activities typically performed during the global period. This is a curated list of many of the common activities described during the post-operative period by physicians/NPPs and other clinical staff:

- review vitals, laboratory or pathology results, imaging, and progress notes

- take interim patient history and evaluate post-operative progress

- assess bowel function

- conduct patient examination with a specific focus on incisions and wounds, post-surgical pain, complications, and fluid and diet intake

- manage medications

- remove stitches, sutures, and staples

- change dressings

- counsel patient and family in person or via phone

- write progress notes, post-operative orders, prescriptions, and discharge summary

- contact/coordinate care with referring physician or other clinical staff

- complete forms or other paperwork.

CMS defines many of the services in this list as "miscellaneous services" included in the global period (e.g., change dressings, remove sutures, change lines) (CMS, 2015).

This selection of activities is not meant to be an all-inclusive list as there is considerable variety in post-operative care. Some activities were more specific for a given type of procedure and/or specialty. For example, ophthalmologists described post-operative care that involved measuring vision, measuring eye pressure, and dilating and refracting eyes. Orthopedic surgeons described post-operative care involving placing and removing casts and examining range of motion. Otolaryngologists described post-operative care that involved reviewing audiograms and tympanometry. Besides face-to-face visits with patients, some interviewees indicated that nonface-to-face care (e.g., talking with a patient via telephone or talking with a patient's family) accounts for a substantial fraction of post-operative care. 


\section{Other Physicians/NPPs and Clinical Staff Involved in Post-Operative Care}

Interviewees described a wide range of physicians/NPPs and clinical staff involved in the delivery of post-operative care, including physicians (e.g., anesthesiologists, hospitalists, intensivists, other proceduralists, primary care providers, and residents), NPPs (e.g., NPs and PAs, audiologists, optometrists, physical therapists, occupational therapists), and clinical staff (e.g., RNs, medical assistants, dietitians, cast technicians). Only eight proceduralists, representing four specialties and primarily working in small private practices, did not report any co-management of patients with other physicians.

Other physicians/NPPs may provide all post-operative care, or work with the proceduralists to co-manage care and comorbidities, or oversee pain management. NPPs and clinical staff were involved in the delivery of post-operative care in a variety of ways including removing sutures and staples, changing dressings, assessing patient progress, reviewing patients' diets, educating patients, and responding to patient phone calls. Most interviewees indicated that care was primarily delivered by the proceduralist, but some indicated that NPs and PAs take the lead in providing post-operative care during office-based visits. One proceduralist reported that he had minimal participation in the delivery of post-operative care, with a resident or PA instead delivering almost all the care. Clinical staff also played an important role in speaking to patients over the phone.

\section{Setting of Care, Work, and Intensity}

When discussing level of work, most interviewees were comfortable describing postoperative visits using the levels of E\&M visits as a framework. However, most felt that the services included in E\&M visits did not fully reflect the scope of post-operative care delivered.

Interviewees generally believed the setting of care could be used to approximate work. Most interviewees believed that work occurred in a setting hierarchy, with the greatest work taking place in the intensive care unit (ICU), then a non-ICU inpatient setting, and finally the office setting. The ICU and inpatient settings typically required more time. There was less consensus on the hierarchy of intensity. (As noted in Chapter 2, intensity refers to a physician's cognitive effort and judgment, technical skill and physical effort, and stress involved in providing care for a patient.) Many believed intensity had the same hierarchy — greatest in the ICU, then non-ICU inpatient setting, and the lowest in the office setting. However, several interviewees disagreed with this hierarchy; one interviewee indicated that all post-operative care was the same intensity, i.e., "easy," regardless of setting. Some believed that office-based care was more intense than inpatient care, primarily when complications emerged. For example, one interviewee indicated the first office visit is more intense than the inpatient post-operative visits because the first office visit includes creating a treatment plan and assessing potential complications, which require greater clinical judgment and stress because the implications of complications may be serious. 
Finally, there was consensus that the majority of post-operative visits were straightforward, but that the work was greater regardless of the setting in those patients who developed complications. Therefore, the post-operative treatment course was atypical.

\section{Contextual Factors in Post-Operative Care}

Interviewees described significant heterogeneity in how post-operative care is delivered across proceduralists and/or practices. Although individual preference drives much of this heterogeneity, several contextual factors appear to influence what is provided including geography, surgical specialty, practice characteristics, and patient characteristics. Several interviewees discussed the role of geography. One proceduralist described that patients sometimes travel from a rural area several hours away for surgery. In these cases, the proceduralist may opt to keep a patient overnight for observation to prevent patients from having to travel back to the hospital in case of an emergent issue. For another proceduralist, one-third of his patients travel from a distant community to a large academic setting for treatment. In this case, office-based post-operative visits are limited at the academic center because of patient travel constraints and patients receiving most routine post-operative care from their local physicians.

In addition, both surgical specialty and practice characteristics affect post-operative treatment. One proceduralist mentioned that some proceduralists may care for less-insured, lessaffluent, and more highly comorbid populations who tend to have higher rates of complications. The makeup of clinical teams may vary greatly from practice to practice. One respondent in an academic practice described a large team of attending surgeons, residents, interns, medical students, pharmacists, NPs, and PAs. Another noted that after surgery in their group, hospitalists provide primary management and address most of the post-operative care.

Physician training also likely affects post-operative treatment provided. One proceduralist suggested that he might be less likely than others to co-manage care with intensivists or hospitalists because he held critical care board certification. Another interviewee suggested that years of training coupled with physician/NPP confidence might reduce "unnecessary" postoperative visits.

\section{Summary of Interview Findings}

Overall, a key theme of the interviews was the heterogeneity in post-operative care. Differences in post-operative care were due to the type of procedure, setting of procedure, geography, and patient, provider, and practice characteristics. Despite these differences, respondents did report some similarity in post-operative care delivered. For example, postoperative care included similar tasks (e.g., taking history, reviewing vitals, checking wounds, and managing pain). In addition, most interviewees agreed that post-operative care in inpatient settings was more work than in office-based settings. Recognizing the differences and 
similarities in the delivery of post-operative care, Chapter 4 describes the possible challenges when capturing such care via claims. 


\section{Challenges Identified During Interviews and Expert Panel in Capturing Post-Operative Care via Codes}

During both our interviews and subsequent expert panel, we identified a number of challenges to capturing post-operative care via codes. In this chapter, we first provide an overview of the expert panel and then an in-depth description of these challenges. Challenges described are related to the work component of post-operative care, the direct costs portion of PEs, and the implementation of nonpayment codes.

\section{Expert Panel}

Following the interviews, we convened an expert panel to further discuss the strengths and weaknesses of options for capturing post-operative care using claims. Results from our interviews were presented to the expert panel to guide discussion. To elicit diverse opinions, we recruited panelists from multiple specialties and backgrounds areas. We identified potential panelists with expertise in claims-based data collection through a snowball approach, based on our knowledge of the field, prior participation in similar panels, and suggestions from interviewees. We included proceduralists who performed a wide range of procedures and who reflected a balance of geography, as well as rural/urban diversity. However, we did not seek specialty society nominations for the panel. We emphasized to panelists that we did not want them to represent their society or specialty; rather we wanted their personal opinions informed by their expertise and experience.

The panel was ultimately made up of nine panelists from six states and the District of Columbia. Most, but not all, of the panelists had some background in health services research and/or payment policy. The panelists included a general surgeon, a dermatologist, an ophthalmologist, an orthopedic surgeon, a cardiothoracic surgeon, an anesthesiologist, a hospitalist, a surgical NP, and a researcher with expertise in the area of post-operative payment. Panelists were offered a $\$ 1,000$ honorarium for their participation, and their travel expenses were covered when applicable.

The panel took place on March 9, 2016, at RAND's office in Arlington, Virginia. First, panelists were briefed on the results of the interviews (as summarized in Chapter 3). Then we had a brainstorming session during which panelists were asked to think of the goals of CMS's data collection on post-operative care using nonpayment claims codes and potential methods for doing so. Different methods of capturing post-operative visits were discussed and panelists were asked to comment on the strengths and weaknesses of those methods. 


\section{Challenges Identified During the Interviews and/or Expert Panel}

\section{Identifying and Capturing Who Performs the Post-Operative Work}

As described in Chapter 2, when the global payments were developed, the premise was that the payment provided for post-operative care was for services performed directly by the proceduralist him/herself. However, in current practice, identifying and isolating which services are attributable to a proceduralist is a key challenge. In particular, proceduralists may work closely with qualified NPPs, hospitalists, intensivists, and anesthesiologists (all of whom may bill independently) and other specialists who manage complicated co-morbidities, as well as residents who furnish post-operative care, but do not bill. This care might occur at a visit during which the proceduralist did not also see the patient, or even outside of a visit (e.g., via telephone).

To properly value both the work and PE components of post-operative care, it is important to understand the services provided during the global period by other physicians/NPPs and clinical staff. The extent to which such physicians/NPPs and clinical staff are substituting or complementing the proceduralist's post-operative activities varies widely. In many casesparticularly for complex patients who have multiple comorbidities - an additional physician such as an intensivist or hospitalist or medical specialist is routinely required to effectively manage the post-operative care. This may be particularly true when such physicians are managing issues unrelated to or pre-dating the procedure (e.g., uncontrolled diabetes, malignancy). However, there are other cases in which another physician/NPP is substituting for a proceduralist's typical post-operative activities. For example, a hospitalist may round on post-operative patients on the floor instead of the proceduralist. Or an intensivist may manage all aspects of the care (e.g., pain management, fluids) except for selected issues such as wound checks. This issue of substitution versus complementarity is not limited to the inpatient setting. Multiple physicians/NPPs and clinical staff also may contribute to the patient's post-operative care in the office setting.

A central question is whether a rule of thumb can define when the involvement of other physicians/NPPs is substituting for a proceduralist and thus should be included in the global post-operative payment. One panelist suggested that a proceduralist could designate himself or herself as providing "primary" management versus "secondary" management. For example, if the proceduralist were leading the ICU team providing post-operative care, he or she would be considered the primary manager, in contrast to a scenario in which an intensivist is leading the care. Such care could be captured differently to indicate the degree of the proceduralist's involvement (e.g., with a primary versus secondary code). Another approach that was discussed by the expert panel was to make this designation of primary and secondary based on the "attending of record."

One issue noted during interviews was how aware or not the proceduralist was of the other work performed during the post-operative period. During many interviews, we needed to prompt the proceduralist to help him or her recall all of the work done by other physicians/NPPs and 
clinical staff. Given the many procedures performed by a given proceduralist and the varying scope of care for each procedure, it is not surprising that the proceduralist may not know all of the care provided. However, this may be an inherent challenge when capturing post-operative care with codes. Several panelists believed that any system of nonpayment codes may be better able to capture work done by the proceduralist him/herself than the work done by clinical staff (e.g., nutritionist).

\section{Heterogeneity in the Scope of Post-Operative Care}

As described in Chapter 3, there is great variation in the scope of the activities performed by proceduralists in the post-operative period. One underlying question is whether the intensity of these different post-operative activities varies and, if so, how this variation should be captured. Some interviewees felt that the intensity of post-operative care was reasonably constant, while others believed that face-to-face activities are more intense than those that can be done non-faceto-face. As noted in the prior chapter, some panelists believed that post-operative care in the ICU was of higher intensity than care provided in the office setting. Similarly, some interviewees and panelists noted that the first visit tends to be much more intense (in addition to lengthier) than subsequent visits. Some interviewees and panelists argued that certain post-operative activities, such as complex ophthalmologic care, require particular surgical skill. If a coding system assumes that work intensity is constant, then it could unfairly reward practices and/or specialties with relatively low work intensity and punish practices and/or specialties with relatively high work intensity.

Many interviewees and panelists noted that the levels of E\&M visits correspond well to the varying levels of post-operative visits, but that the services included in E\&M visits do not reflect the diversity of post-operative care. Moreover, they noted that the documentation requirements for E\&M visits can be substantial and that such requirements would put an undue burden on proceduralists as they were not accustomed to documenting the necessary elements for $\mathrm{E} \& \mathrm{M}$ visits during their post-operative visits.

Many interviewees also described providing post-operative care outside of an in-person visit, or non-face-to-face care. Such care can be categorized as occurring on the day of the visit or on a day other than that of the visit. Non-face-to-face time on the day of the visit could include the proceduralist communicating with a variety of other physicians/NPPs (e.g., oncologists, infectious diseases specialists), as well as nonbilling clinical staff to review diagnoses and adjust management. Such time also includes reviewing laboratory results, pathology reports, imaging, and other services' notes. This type of synchronous non-face-to-face care is most often an issue with inpatient care, but is also present in office-based visits. Non-face-to-face care on a day other than that of the visit could include such activities as answering secure email messaging via online patient portals (Crotty et al., 2014) and coordinating care with other specialties (particularly in the office-based setting). 
During the expert panel there was discussion of the value of having a unique code for nonface-to-face care (with the underlying assumption that there are key differences in the intensity and scope of care provided in such care). The advantage of such an encounter code is that it would allow CMS to capture the scope of this care at a more granular level and, as discussed in Chapter 3, many proceduralists believe that much of their post-operative work is provided outside a face-to-face visit. The disadvantages are the additional complexity of having another code, the concern that current billing systems may not have the capacity for physicians to submit such codes, and that electronic medical records (EMRs) may not be set up to handle such encounters, thus limiting the ability to document these non-face-to-face encounters (to validate that they occurred). Several panelists noted that non-face-to-face care is common with an E\&M visit. Therefore, it was not clear to them that it was critical to incorporate non-face-to-face care with post-operative visits.

\section{Categorizing the PE Component of Services}

There are a number of considerations when accounting for the direct PE component of postoperative care. The PE component of a post-operative visit for clinical staff time, specific supplies, and equipment is taken into account in the valuation of the global package, and often exceeds the PE associated with an E\&M visit.

Activities performed by the clinical staff versus the proceduralist vary by specialty, procedure, and individual practice (depending on the structure and style of the practice). Perhaps most challenging is the relationship between the proceduralist's activities and the clinical staff's activities. Many panelists felt there was a clear correlation between the proceduralist's work and clinical staff's work - such that if a proceduralist does more work, so too does the clinical staff. However, it is feasible for a proceduralist to shift as much of his or her post-operative work as possible to clinical staff, resulting in less work for the proceduralist and an inverse relationship to clinical staff work. These competing issues may make it challenging to capture the component of PE related to clinical staff solely through codes for the proceduralist's work. While the panel recognized these issues, the consensus was that this may be more of a theoretical concern and that the complexity of trying to capture variation in clinical staff time in the context of an inperson visit with the proceduralist is likely not worth the effort.

Additional PEs - including clinical staff — are the equipment and supplies used for a visit. Panelists dismissed the idea that there was a direct correlation between physician work and equipment and supplies. While some equipment and supplies may be common across many visits, some procedures may require unique equipment and supplies. For example, ophthalmologists may require specialized and expensive equipment both for in-office procedures and follow-up visits that would not be used by any other specialties. Many proceduralists - both interviewees and panelists - pointed out that the RUC has done an immense amount of work to value PE via the AMA's Practice Expense Advisory Committee (PEAC) (now the AMA RUC 
Practice Expense Subcommittee), and it was better to build on this prior work rather than use a new set of claims codes to capture these PEs.

\section{Low Variability in the Complexity of Post-Operative Visits}

In both the interviews and expert panel, we heard that the vast majority ( 90 percent was often specifically cited) of post-operative visits are straightforward. Only a small percentage of visits are of a complex nature. The challenge is how to capture these exceptions. This echoes the AMA's assertion that the vast majority of post-operative office visits are CPT codes 99212 and 99213, both of which represent lower complexity E\&M visits (Table 4.1). Table 4.1, created by the AMA, reports the 2015 global surgical E\&M utilization percentage by CPT code. These percentages were calculated using expected counts of the number of the post-operative visits per CPT code, available from the CMS website and the RBRVS Data Manager database, which are based on surveys of physicians conducted by specialty societies and reviewed by the RUC and CMS to estimate the number and total time of visits. These expected counts of post-operative visits per CPT code were volume-weighted based on the corresponding count of total 2015 Medicare claims data per CPT code. These percentages reflect all procedures, specialties, and physicians included in these databases (Morrow and Smith, 2016).

Table 4.1. 2015 Global Surgical E\&M Utilization Percentage by CPT Code

\begin{tabular}{cc}
\hline CPT Code & 2015 Global Surgical E\&M Utilization Percentage \\
\hline Outpatient E\&M & \\
99211 & $0.30 \%$ \\
99212 & $57.93 \%$ \\
99213 & $40.67 \%$ \\
99214 & $1.07 \%$ \\
99215 & $0.03 \%$ \\
& \\
\hline Inpatient E\&M & \\
99231 & $56.30 \%$
\end{tabular}


99232

99233

99291
$30.45 \%$

$10.06 \%$

$3.19 \%$

SOURCE: AMA.

\section{Incentives for Proceduralists to Submit Nonpayment Codes}

A common issue addressed in both interviews and the expert panel discussion was the lack of incentives for physicians/NPPs to submit nonpayment codes. While some interviewees and panelists reported that they or their colleagues submit the current nonpayment 99024 code for their post-operative visits (often because their EMR required physicians to submit a CPT code for each visit), many interviewees stated they currently provide minimal documentation for their post-operative visits and did not submit any type of claim. Proceduralists are not used to billing for inpatient hospital visits other than as a consulting physician because of the global period. Submitting new post-operative visit codes, on average, will increase physician/NPP workloads and potentially disrupt their workflows. Therefore, panelists were concerned that this would lead to substantial underreporting of visits. While physicians/NPPs will likely recognize the importance of reporting on their post-operative care for their specialty and future income, there is little direct negative impact of not submitting such a claim for data collection purposes. Underreporting could be exacerbated if the necessary documentation to support the new nonpayment codes is judged to be excessive. In addition, physicians/NPPs might not report a visit or err on the side of using an inappropriately lower-level visit because of the fear of an audit violation. Panelists also pointed out that the external coding companies that many proceduralists and hospitals use may not prioritize nonpayment codes given that one key goal of these companies is to maximize revenue.

There were also competing concerns about overreporting of post-operative visits. Specifically, in an effort to maximize payment for procedures, one panelist believed proceduralists will bring patients back for more visits than are necessary during the reporting period or inappropriately record visits using a code for a higher-level visit.

Under MACRA, Congress dictated that CMS can potentially withhold up to 5 percent of payments to physicians and practices who do not participate in code submission and subsequent documentation. The panelists and interviewees consistently believed that 5 percent was too large of a withhold.

Another common concern that emerged from the interviews and the panel was the educational burden of introducing a new set of nonpayment claims codes. One panelist even 
suggested that given the enormity of the task in such a short time frame, accurate data collection via claims would be "impossible." Above all, the interviews and expert panelist discussions consistently emphasized the need for simplicity in any coding system.

\section{Summary of Challenges Associated with Capturing Post-Operative Care}

Both the interviews and the expert panel identified multiple challenges in capturing postoperative care, including: (1) who performs the work provided (proceduralist versus nonproceduralist physician versus NPP), as well as the heterogeneity of the work itself; (2) how direct costs related to PEs, such as staff time, space, equipment, and supplies, should be captured; (3) only a modest proportion of patients requiring visits that were more complex; and (4) the need for a reporting system with a burden low enough so as not to discourage participation. In the context of these numerous challenges, Chapter 5 outlines the strengths and weaknesses of common approaches to capture post-operative care. 


\section{Options for Capturing Post-Operative Care}

As detailed in Chapter 4, there are a number of challenges that must be addressed in creating a set of claims-based codes to capture post-operative care. In this chapter, we describe the strengths and weaknesses of four approaches for capturing post-operative care through claims based on: (1) setting of care; (2) existing E\&M codes; (3) scope of care; and (4) time.

\section{Setting of Care}

Some interviewees and panelists believed that site of care was a rough proxy for work. For example, caring for a patient in the ICU is typically more work than caring for a patient in an office setting. Given that setting is a critical driver of work, capturing the level of a visit could be addressed by the setting in which it occurred. One potential system of post-operative codes could consist of three levels: (1) ICU post-operative visit; (2) non-ICU inpatient visit; and (3) office visit. An additional fourth level could be added, such as an inpatient step-down unit that would be between ICU level and inpatient ward level of care. Proceduralists would code the postoperative visit based on the setting in which they saw the patient.

The strength of this approach is a lower burden of work, as it is straightforward for clinicians to know which code to use. Thus, education on coding would be relatively simple.

Documentation might be easier. Potentially the greatest weakness of this approach is that both interviewees and panelists believed there was great heterogeneity within post-operative visits in a given setting. Therefore, such a system would not fully capture variation in the level of postoperative care. Moreover, specific specialty societies may feel that they have above average work in certain settings of care, so such a system would unfairly penalize these specialties. Other weaknesses of this approach are that other factors might dictate what setting of care is provided. For example, ICU utilization may be driven by factors other than patient need, such as ICU-bed availability (Gooch and Kahn, 2014). Finally, such a system would not capture whether the proceduralist is providing primary management versus secondary management (e.g., intensivist is providing most of the care with a proceduralist co-managing the care).

\section{Existing E\&M Codes}

Most proceduralists are very familiar with using E\&M visit codes. The E\&M section of the CPT code set is divided into four broad categories: (1) office visits, (2) hospital visits, (3) consultations, and (4) non-face-to-face services. Most of these categories are then further divided into two or more categories (e.g., new patient versus established patient, initial versus subsequent hospital visit). Subcategories are then classified into levels with specific codes (e.g., level 1-5 for office-based visits, or level 1-3 for inpatient visits). These codes attempt to capture 
complexity based on: (1) the number of possible diagnoses/management options; (2) the amount of complexity of medical records, diagnostic tests, and/or other information to obtain, review, and analyze; (3) risk of significant complications, morbidity, mortality, and comorbidities; (4) diagnostic procedure(s) and possible management options; and/or (5) the time required to provide the specific service.

These same codes could be used to capture post-operative visits. For example, there are currently three levels of noncritical care inpatient follow-up visits. For a follow-up postoperative visit in the inpatient setting, the proceduralist would choose the correct visit based on complexity and then submit a code for this visit. Proceduralists could use a new modifier code to indicate this was for data collection and not for payment.

The main strength of using E\&M codes is that they are familiar to most clinicians. This is a key issue given the short timeline and logistical constraints of implementing this data collection system. The breadth of E\&M codes also allows proceduralists a wide range of possible codes from which to choose. Finally, the E\&M codes are currently used by the RUC in valuation of post-operative work.

However, panelists and interviewees identified a number of challenges with using the E\&M framework to capture post-operative work, including:

1. Excessive documentation requirements. Possibly the greatest concern raised by the panelists was that using the E\&M structure would require that proceduralists support the code selected in their documentation. Given that proceduralists currently use minimal documentation when they have a post-operative visit, using the E\&M structure would place a substantial administrative burden upon proceduralists. One panelist said that if the E\&M framework was used, it would be critical to waive the documentation requirements of E\&M visits. However, if documentation were to be waived, then concerns could be raised afterward about the use of inappropriate codes.

2. Overly complex. Among all of our interviews and panel discussions, simplicity was noted as the single most important factor in choosing a system to capture post-operative care. E\&M codes are complex, with at least ten codes for office visits and at least 12 different codes for inpatient visits (if including new visits). The list of codes is longer when codes for non-face-to-face visits are included. Each code has detailed and extensive history and exam requirements. A system that used fewer codes for data collection may be preferable.

3. Use of an existing flawed system. The E\&M system is felt to be a generally flawed way to capture visit complexity (Berenson, Basch, and Sussex, 2011; Berenson and Goodson, 2016; Brett 1998). As one critic argued,

To determine the appropriate billing for a general evaluation or management service under the new guidelines, one must follow five steps: count how many of eight approved elements of a medical history are documented in the history of present illness, count how many of 14 approved body systems are documented for a 'review of systems'; count how many elements of the medical history, family history, and social history are documented; count how many of 14 approved organ systems or body areas are documented for the physician examination; and count how many approved physical-examination maneuvers are 
documented within each examined organ system. (Berenson, Basch, and Sussex, 2011).

This has led to numerous problems, including overdocumentation, upcoding, and undercoding (Berenson, Basch, and Sussex, 2011).

4. May not be well suited to capture what drives complexity in post-operative visits. As noted earlier, E\&M billing requirements are built upon complexity in such elements as the medical history, review of systems, family history, social history, family history, and how many organ systems are examined. In the context of a post-operative visit, many of these elements may be irrelevant. For example, a proceduralist may only need to examine the wound site when addressing a potential post-operative infection. Using the E\&M structure might also drive proceduralists to ask irrelevant questions. When an orthopedic surgeon does a routine post-operative wound check after rotator cuff repair, using an E\&M framework's need for "highly complex" history of present illness, the surgeon would need to describe the location, quality, severity, duration, timing, context, modifying factors, and associated signs or symptoms. These questions may not be necessary in the post-operative management of the rotator cuff.

5. Potential for confusion. Because the E\&M structure may not be well suited to postoperative care, several panelists suggested using the same codes in current use (e.g., 99213), but providing supplemental definitions specific to the post-operative context. However, other panelists expressed concern that using the same E\&M codes with different definitions might create substantial confusion among proceduralists. Furthermore, if modifier codes were used, proceduralists could erroneously submit an E\&M visit by forgetting to submit the appropriate post-operative modifier code. Overall, panelists supported codes that were structured similarly to E\&M codes, but accurately reflected the care delivered during post-operative visits and had lesser documentation requirements.

\section{Scope of Care}

Scope of care represents what care is provided in the post-operative visit. Because the majority of post-operative visits represent straightforward care (as discussed in Chapter 4), the key in using scope of care to define level of post-operative visits is how to capture the small fraction of visits with more work.

One high-level framework discussed at the expert panel consisted of two types of office visits: (1) a basic post-operative visit that would address common post-operative issues, including evaluation of interim progress, removing stitches, or changing dressing; and (2) a complex post-operative visit that would include management of complications, such as wound infections, and complex care, such as removing sutures from within the eye. The framework was discussed at the expert panel with the idea that at least 80 percent of post-operative visits would be the basic type.

The strength of such an approach is that it uses the actual care provided to decide on the level of visit. In contrast, site of care is only a rough proxy for the level. If a small number of codes are used, then it could be potentially straightforward for proceduralists to choose the relevant code. 
However, the main challenge to this approach is that there is an enormous breadth of postoperative tasks. Categorizing all of those services into basic or complex could be challenging and controversial given the time constraints associated with implementation of the codes. If more than two levels are created to increase granularity of the data collected, then the challenge of categorizing care could prove to be even more difficult.

\section{Time}

As noted in Chapter 3, some interviewees believed that intensity of post-operative care is constant, and that the variation in work for post-operative visits was primarily driven by the time required to provide care. The current E\&M framework has many examples of time-based codes (e.g., discharge service, critical care, and prolonged services). Therefore, one possible way to capture post-operative visits would be via similar time-based codes.

At the expert panel meeting, we discussed a framework with three visit levels: (1) basic visit, 0-10 minutes; (2) intermediate visit, 11-20 minutes; and (3) complex visit, 21-30 minutes. Time could be based on face-to-face time with a patient or include other non-face-to-face tasks (e.g., reviewing imaging studies).

The strength of this approach lies in its simplicity and, therefore, a lower educational burden for proceduralists. Also, if time is the main driver of work then it better captures level than site of care. Moreover, time may be relatively easy to document. The main weakness is the assumption that there is a constant intensity across different specialties, settings, and services. Many panelists felt uncomfortable with this assumption. A related consideration would be deciding which aspects of time should be included (i.e., face-to-face versus non-face-to-face time). Another potential weakness is that proceduralists are not used to billing time-based codes, and thus this approach may be unfamiliar. Finally, panelists raised concerns that encouraging physicians/NPPs to take longer to provide post-operative care might create incentives for inefficiency.

\section{Summary of Options}

In this chapter, we discussed the strengths and weaknesses of using site of care, E\&M visits, scope of care, and time to capture post-operative care. It is clear that none of these approaches alone is unequivocally superior. While site of care is simplest, it suffers from lack of granularity as specific sites contain significant heterogeneity. E\&M is familiar to proceduralists, but the codes are a poor fit for the unique circumstances of post-operative care and could lead to considerable documentation burden. Scope of care is potentially the most granular, but determining which service from the myriad of services fits into which category of care may be difficult. Time is likely the easiest to understand for proceduralists, but it is limited by the notion that not all proceduralists' times are created equal. Importantly, these approaches are not mutually exclusive. In Chapter 6, we present a hybrid approach. 


\section{Recommendations for Capturing Post-Operative Care}

In considering the input from interviews and the expert panel, several essential considerations for the reporting of post-operative care using claims emerged. The first is the importance of devising a simple system to facilitate reporting. The second is that the vast majority of post-operative visits are straightforward. Therefore, the key is identifying the smaller number of complex visits, which may be associated with a select number of procedures. The third is not to use the E\&M structure to capture post-operative care because, as explained earlier, E\&M codes are inadequately designed to capture the full scope of post-operative care and using such codes might create confusion. Fourth, no single framework (site of care, time, or scope of care) is ideal for capturing post-operative visits. The expert panel was most enthusiastic about a set of codes that used elements of all three frameworks. Fifth, it is important to distinguishparticularly in the inpatient setting - between circumstances where a proceduralist is providing primary management of a patient versus secondary management. Sixth, nonpayment codes are a poor mechanism to capture PEs, which should be captured by other means. Seventh, much postoperative care occurs outside of in-person visits and by clinical staff - so it is important to create a mechanism to capture this care if the resources required are to be considered.

\section{Proposed Framework of Post-Operative Codes}

Based on these considerations, we propose the following eight codes for capturing postoperative visits (Figure 6.1). Six of these codes would be used by physicians and NPPs, while the other two are specific to clinical staff. Each of these codes represents a ten-minute increment of care. The remainder of the chapter explains the codes in further detail. 
Figure 6.1. Proposed Post-Operative Visit Codes ${ }^{a}$
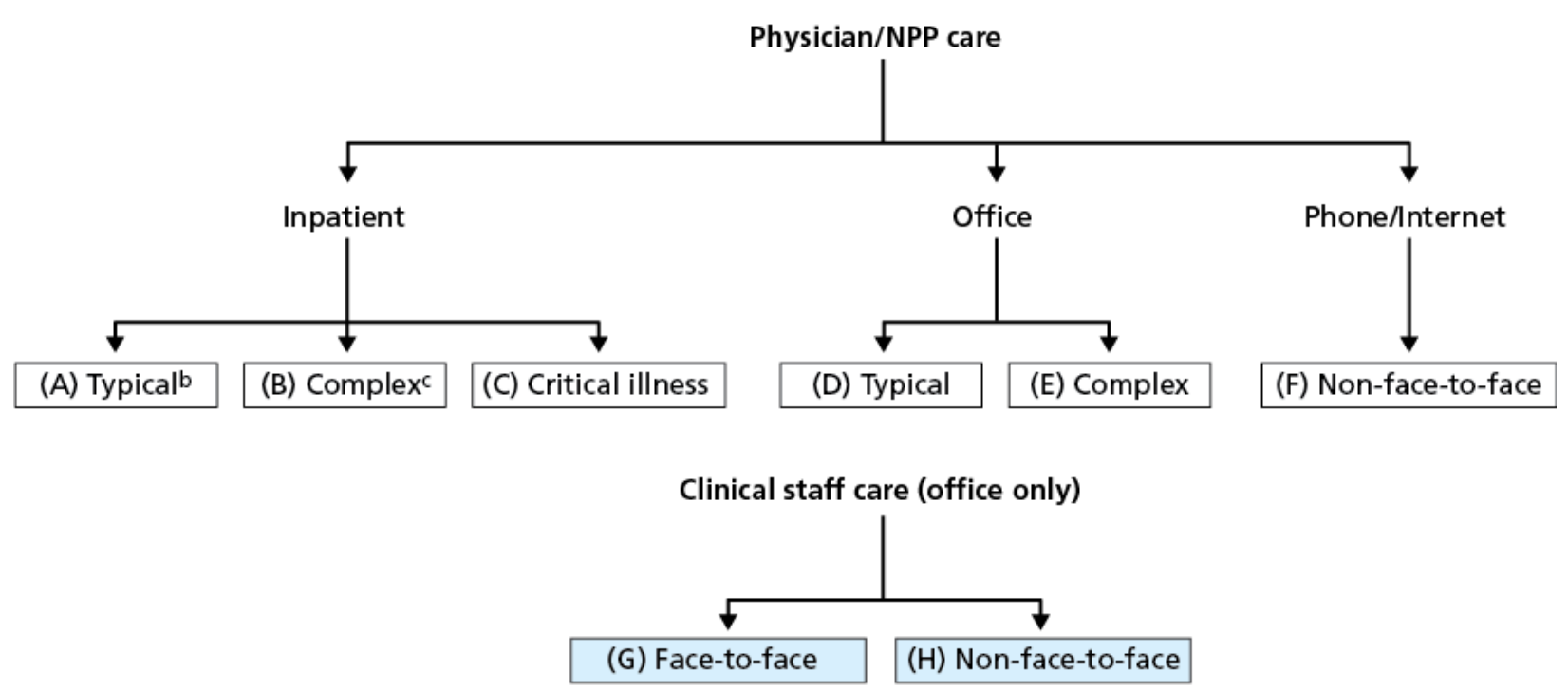

a Each code represents a ten-minute increment of care.

b Typical visits include any of the most common services provided during postoperative visits (e.g., wound checks, dressing changes) and are described in more detail later in this chapter.

' Complex refers to visits that go beyond typical visits (and do not fall under the critical illness category), such as special postoperative diagnostic testing or procedures, and are also described in more detail later in this chapter. RAND RR1526-1

\section{Inpatient Codes: $A, B$, and C}

As noted earlier, the panelists were most enthusiastic about a system that combines (1) setting of care, (2) scope of care, and (3) time-based care. In the inpatient setting, we propose three codes based on scope of care: (1) typical inpatient post-operative visit; (2) complex inpatient post-operative visit; and (3) critical illness visit. The assumption is that the typical inpatient visit would be used for the majority of visits and the complex and critical illness visits would be used less frequently. Time would be captured in ten-minute increments (how time is measured is discussed in more detail later in this chapter). Time increments add a layer of granularity to the codes without adding a substantial burden of unnecessary complexity.

The typical inpatient visit would be used when any of the following services are provided:

- Review vitals, laboratory or pathology results, imaging, and progress notes

- Take interim patient history and evaluate post-operative progress

- Assess bowel function

- Conduct patient examination with a specific focus on incisions and wounds, post-surgical pain, complications, and fluid and diet intake

- Manage medications (e.g., wean pain medications)

- Remove stitches, sutures, and staples

- Change dressings 
- Counsel patient and family in person or via phone

- Write progress notes, post-operative orders, prescriptions, and discharge summary

- Contact and coordinate care with referring physician or other clinical staff

- Complete forms or other paperwork.

The highest level of inpatient visit would be a "critical care post-operative visit." The definition of critical illness would build upon the current definitions used in CPT of critical illness, which requires acute impairment of one or more vital organ systems and a high probability of imminent or life-threatening deterioration in the patient's condition. However, our definition of critical illness contrasts with the CPT definition in two important ways. First, our definition emphasizes that it should only be used when the proceduralist is providing primary management of the patient. This would help address circumstances where the proceduralist remains actively involved, but an intensivist is providing primary management. The second difference is the difference in time increments. Current CPT critical illness codes use longer time elements. We are proposing ten-minute increments to be consistent with the other post-operative visit codes.

If a case is more complex than the typical visit, but is not a critical care visit, then that visit would be coded as a "complex inpatient post-operative visit." Some potential circumstances that might merit the use of a complex visit include: (1) secondary management of a critically ill patient where another provider, such as an intensivist, is providing the primary management but the proceduralist remains actively involved; (2) primary management of a particularly complex patient such as a patient with numerous comorbidities or high likelihood of significant decline or death; (3) management of a significant complication; and (4) complex procedures outside of the OR (e.g., significant debridement at the bedside). The definition of this visit would be based on the judgment of the proceduralists and the expectation is that the physician/NPP will document in a single line what they did in this post-operative visit and that in their judgment it goes beyond a typical post-operative visit.

In defining complex visits based on the judgment of the provider, we are addressing concerns of numerous interviewees and technical panelists that creating a list of specific selected services that qualify as "complex" would be potentially arbitrary and infeasible. Moreover, a brief justification addresses the concerns about excessive documentation that arose around using the E\&M system to capture post-operative care (discussed in more detail in Chapter 5). We trust that proceduralists would provide a simple, brief justification for why their visits go beyond typical post-operative care (e.g., "I performed a complex removal of sutures that I believe goes beyond typical post-operative care"). Importantly, such medical note documentation may also serve to potentially inform future iterations of this new coding framework.

For each of the three types of inpatient codes, physicians/NPPs would bill their time in tenminute increments. We recommend using a time estimate that includes non-face-to-face time, including care coordination and reviewing records, as interviewees stressed that these activities 
were common post-operative activities, particularly in the inpatient setting. Including non-faceto-face time would be familiar to proceduralists who provide critical illness care as current CPT guidelines for such care also incorporate non-face-to-face care. Thus, these ten-minute increments would be based on aggregate time spent on that calendar day in the patient's room, at the nursing station, or on the inpatient ward for activities, including

- $\quad$ spending time with patient, family members or surrogate decisionmakers, taking medical history, performing a physical exam or other care (e.g., debridement), reviewing the patient's condition or prognosis, or discussing treatment

- reviewing test results or imaging studies

- discussing patient's care with other medical staff

- documenting care.

As noted in Chapter 5, excessive documentation requirements might lead to underreporting. To increase simplicity, documentation in the medical record for a typical inpatient office visit could be, "I spent 18 minutes in typical post-operative care." Documentation for a complex visit could be, "I spent 25 minutes providing , which I judged to be beyond typical postoperative care."

CMS would need to establish rules regarding who should report services provided within the same group practice that are considered part of the global package. For data collection purposes, for example, the expectation might be that the proceduralist or physician/NPP providing the postoperative care would use their own Medicare billing code to submit the post-operative visit code. If both the proceduralist and NPP provided care during a single visit, then the code would be submitted by the physician based on the combined time of the qualified NPP and the physician. (Note: Based on current CPT rules, only physician time is used for critical care. For these postoperative visits, we recommend combining physician and NPP time.) In addition, CMS would need to address the reporting rules for when a resident provides care under the supervision of a teaching physician.

\section{Office-Based Visit Codes: D and E}

Office visits are divided into two major categories: (1) typical post-operative office visit; and (2) complex post-operative office visit. Typical office-based post-operative visits would cover the same list of activities as a typical inpatient post-operative visit, except they would not include discharge planning or discharge summaries.

A complex office visit would include care that is more complex than is covered in a typical visit. Similar to the inpatient codes, the expectation is that this would be used relatively infrequently. Potential circumstances might include: (1) management of a particularly complex patient, such as a patient with numerous comorbidities or high likelihood of dying; (2) management of a significant complication; or (3) management or discussion of a complex diagnosis (e.g., new cancer diagnosis, high risk of mortality). Again, the decision to use the 
complex visit code would be based on the judgment of the physician/NPP. The physician/NPP would be asked to document the provided care and describe how it went beyond a typical postoperative visit as for the inpatient code B for a complex visit (see code B section).

The key difference between the inpatient post-operative codes and the office post-operative codes is that we recommend the time increments include only face-to-face care for office visits. In the office setting, we believe it will be easier for proceduralists if we mirror the $\mathrm{E} \& \mathrm{M}$ traditional framework of using face-to-face time as this is already well understood by physicians/NPPs as explained in the interviews and expert panel. Moreover, interviewees suggested that time required for activities, such as care coordination and reviewing tests and imaging, were less of an issue in the office setting compared with the inpatient setting. Finally, ambulatory care tends to have non-face-to-face time on a day other than that of a visit, when compared with inpatient care. Therefore, while it is feasible to conceptually couple non-face-toface time with face-to-face time in the inpatient setting (e.g., review data, labs, then see patient, talk with family afterward, etc., all happens on the same calendar day), face-to-face time is often uncoupled from non-face-to-face time in the outpatient setting. For example, if the proceduralist sees the patient on Monday, but then calls the patient on Thursday and has an extensive phone conversation about a lab test result, this would be challenging to capture in a single time element.

We recognize that the difference in how time is measured (face-to-face time for office-based care and face-to-face plus non-face-to-face care for inpatient codes) adds a layer of complexity to the proposed coding system. An alternative model for CMS to consider would be one in which time is only measured via face-to-face time for all post-operative care. Using only face-to-face time has the advantage of simplicity, but it has the disadvantage that CMS would not be able to capture circumstances in which a large amount of non-face-to-face time is provided during an inpatient post-operative visit. ${ }^{4}$

Documentation would be similar to that for the inpatient codes, but would be focused on face-to-face time only. For example, a physician/NPP might document, "I spent 18 minutes in face-to-face time with the patient providing typical post-operative care."

\section{Code for Non-Face-to-Face Care: $\mathrm{F}$}

Given the evolution of the health care system and how post-operative care is provided, we propose an additional code for post-operative care provided via phone or Internet modality such

${ }^{4}$ If CMS wanted to use only face-to-face time to measure post-operative work but also capture circumstances in which excessive non-face-to-face time was provided, a ninth post-operative code could be created. This ninth code would be used by physicians/NPPs when they spent excessive non-face-to-face time during an encounter. 
as a patient portal. ${ }^{5}$ This would be a code for care provided by a physician/NPP. Time would be based on time interacting with the patient or composing a message to the patient.

To distinguish between care provided within the context of an in-person post-operative visit (e.g., follow-up of a lab test ordered at a visit), we recommend that this code cannot be used on the same day or in the two days before or after an in-person visit. Of note, most physicians may not be accustomed to billing for non-face-to-face care, so there is a possibility that this code will be underreported.

\section{Codes for Clinical Staff (Office Only): G and H}

In order to account for changes in the health care system and the increasing role of teambased care in post-operative care, we created two additional codes to capture work provided by nonbilling clinical staff. The code would be submitted under the supervising physician/NPP's name per CMS guidelines. It is unclear how often these codes would be used, but they were included for circumstances where clinical staff commonly provide post-operative care. In the office visit setting, time would be defined by the clinical staff's face-to-face time with the patient. In the phone/Internet modality code, time would be defined by calculating the time interacting with the patient or composing a message to the patient.

\section{Choosing the Correct Time Increment}

We held several discussions on the appropriate amount of time to use for a single increment of post-operative care. The goal of this data collection is to give CMS the necessary data to value post-operative work for procedures with a global payment. We recommend ten-minute increments because it was felt to be granular enough to accurately value physician work. A longer time increment, such as 20 minutes, might be problematic in that both a four-minute visit and a 19-minute visit would use the same code and, therefore, create issues when the collected data are used for valuation. An additional advantage is that the number ten is simple and easy to remember. A shorter increment, such as five minutes, might increase the response burden; for example, a visit consisting of 50 minutes would require more than eight codes (or units of service) to be submitted, though the actual incremental burden to do so may not be high.

Using methods published previously (Mafi et al., 2013, Mafi et al., 2015, Mafi et al., forthcoming), we conducted an analysis to evaluate the visit length of pre-operative and postoperative office-based visits for Medicare patients from 1997 to 2012 based on data from the National Ambulatory Medical Care Survey (NAMCS), a nationally representative survey that collects data on the provision and use of nonfederal ambulatory medical care services in the

\footnotetext{
${ }^{5}$ If post-operative care was provided via a live videoconference, we recommend that physician/NPP use the GT telemedicine modifier with the appropriate inpatient or office-based post-operative visit code.
} 
United States (see http://www.cdc.gov/nchs/ahcd.htm for more details) (Centers for Disease Control and Prevention, 2015). The duration of the visit is defined as only the amount of time the physician spent in face-to-face contact with the patient, and does not include time spent with NPPs or clinical staff. Overall, mean visit duration was 17.3 minutes and the median visit length was 15 minutes per visit. Visit length did not increase over time (Table 6.1). ${ }^{6}$ Based on the time range for post-operative visits we observed in NAMCS, we believe that a ten-minute increment for post-operative visits would allow CMS to distinguish between shorter and longer postoperative visits.

Table 6.1. Visit Length Over Time from 1997 to 2012 for Medicare Patients

\begin{tabular}{lllllllll}
\hline Year & $\mathbf{1 9 9 7 -}$ & $\mathbf{1 9 9 9 -}$ & $\mathbf{2 0 0 1 -}$ & $\mathbf{2 0 0 3 -}$ & $\mathbf{2 0 0 5 -}$ & $\mathbf{2 0 0 7 -}$ & $\mathbf{2 0 0 9 -}$ & $\mathbf{2 0 1 1 -}$ \\
\hline & $\mathbf{1 9 9 8}$ & $\mathbf{2 0 0 0}$ & $\mathbf{2 0 0 2}$ & $\mathbf{2 0 0 4}$ & $\mathbf{2 0 0 6}$ & $\mathbf{2 0 0 8}$ & $\mathbf{2 0 1 0}$ & $\mathbf{2 0 1 2}$ \\
\hline Sample n & 219 & 307 & 122 & 85 & 109 & 89 & 95 & 135 \\
$\begin{array}{l}\text { Mean Visit } \\
\text { Length }\end{array}$ & 17.5 & 18.6 & 17.8 & 13.8 & 15.2 & 17.3 & 18 & 17.1 \\
\hline
\end{tabular}

SOURCE: Authors' analysis.

Incorporating rounding, the ten-minute time increments used in this structure for a typical post-operative visit would be

$\leq 15$ minutes - code $(\mathrm{A}, \mathrm{B}, \mathrm{C}$, etc. $) \times 1$

16-25 minutes - same code as above $\times 2$

26-35 minutes - same code as above $\times 3$

36 minutes or longer - same code as above $\times$ the appropriate number for the length of visit.

As a concrete example, if a proceduralist saw a patient in the office for a typical postoperative visit and spent 18 minutes with them in face-to-face time, he or she would bill the D code twice.

One alternative to ten-minute increments is a system based on one-minute increments. Such a one-minute time increment framework has as precedent among anesthesiologists, who report the

\footnotetext{
${ }^{6}$ We recognize that studies suggest that the NAMCS overestimates office visit length when compared with real-time observation of physician office visits (Gottschalk and Flocke, 2005; Gilchrist et al., 2004). For example, Gilchrist et al. found that the average face-to-face visit duration recorded by direct observation was 12.8 minutes, compared with 16.5 minutes (Gilchrist et al., 2004). Additionally, Gottschalk and Flocke showed that average face-to-face patient time recorded by direct observation was 10.7 minutes, compared with 18.7 minutes as noted in the NAMCS 2003 data file. Nonetheless, we felt the NAMCS estimates were the most appropriate comparison as we are also proposing a self-reported measure of visit length.
} 
exact time in minutes of an encounter on the claim to CMS (CMS, 2007). A similar system for post-operative care might be simpler for proceduralists, who would document the exact length of time for a service (e.g., "I spent 17 minutes providing typical post-operative care") and submit the corresponding units of service (e.g., 17 one-minute codes or 1.7 ten-minute increment codes). It might be simpler as a physician/NPP would not need to round to determine the number of codes to submit. Because the data would provide more granular data, an additional benefit would be facilitating more accurate valuation of post-operative care. We did not propose this oneminute system in our main recommendations because it may not be logistically feasible. We were unsure whether the billing systems and/or coding companies in current use by proceduralists would allow the use of one-minute increment codes. Although one-minute time increments deserve further exploration, such questions would be critical to answer before moving forward.

\section{Summary of Recommendations}

In this section, we proposed a set of eight codes that capture differences in level among postoperative visits using time, setting, and the care provided. In the inpatient setting, visits are divided into typical, complex, and critical care encounters. In the office-based setting, visits are divided into typical versus complex encounters. In both settings, typical visits represent the majority of services, and complex visits allow for some flexibility by acknowledging that some visits do not precisely fit the well-defined typical or critical illness rubrics. Proceduralists have the option of adding extra ten-minute allotments of time in case visits take longer, and we have also allowed for the ability to code for nonbilling clinical staff and Internet- or phone-based care delivery. Importantly, these codes are specifically designed for post-operative care and require fewer visit codes than the current E\&M framework. Moreover, given the need to capture visit complexity and the strong recommendations from the panelists, incorporating time into the coding framework adds a potentially simple and accurate way to capture visit complexity. The recommended codes attempt to balance the need for a simple and straightforward system with the need for a set of codes to capture the granularity and heterogeneity associated with postoperative care delivery. 


\section{Summary and Next Steps}

In Section 523 of MACRA, Congress directed CMS to develop and implement a process to gather the necessary data to appropriately value post-operative care. This data collection should begin no later than January 1, 2017, and the collected data should include both the number and level of medical visits furnished during the global period. Congress suggested this be reported on claims, but it provided flexibility to CMS.

Our objective in this project was to assist CMS by proposing a set of nonpayment billing codes that proceduralists could use to report on both the number and level of post-operative care they provide. In describing the level, it is important to capture the major differences in work and intensity of post-operative care provided during the global periods.

We conducted 33 interviews with proceduralists and other stakeholders on the type of care they provide in post-operative visits and their general thoughts on how best to use such billing codes to capture post-operative care. These interviews were supplemented by a one-day expert panel in which we discussed the challenges in creating such a system and considered the strengths and weaknesses of different approaches.

Several important themes emerged from the interviews and expert panel. First, it is important to create a system as simple as possible. Second, the majority of post-operative visits are straightforward. Therefore, the key is identifying the smaller number of more-complex visits. Third, using the existing E\&M structure may be a poor fit for capturing post-operative care. Fourth, a coding system that combines site of care, time, and scope of care might be best for capturing post-operative visits. Fifth, it is important to distinguish between care when a proceduralist is providing primary management of a patient versus secondary management. Sixth, nonpayment codes are a poor mechanism to capture PEs. Finally, a large fraction of postoperative care is provided outside of in-person visits and by clinical staff; thus, it is important to create a mechanism to capture this care.

We recommend a total of eight codes for different types of post-operative care based on both the setting and the provided care. Three codes are for inpatient care, three codes are for office visits, and two codes are for care provided by telephone or electronically. Two of the codes are specific to care where there is no physician/NPP interaction. Each type of the eight codes is used to capture 10 minutes of post-operative care, and physicians/NPPs would be expected to submit codes for additional increments for longer visits.

\section{Other Considerations}

The interviewees and panelists discussed other issues important to consider when capturing post-operative care, but they are outside the scope of this project. One particularly important 
issue is which proceduralists will use the post-operative visit codes. Will it include all proceduralists or a smaller representative sample? For example, if all proceduralists are expected to use the codes, simplicity is a particular concern to minimize educational costs. If a smaller set of proceduralists uses the codes, then a more-complicated system may be feasible. A large sample of proceduralists may be necessary as the interviewees stressed there is great variation in how post-operative care is provided in rural and urban settings, large versus small practices, academic medical centers versus private offices, across different regions, and by specialty.

Interviewees and panelists noted that the short time frame for implementing a system scheduled to start on January 1, 2017, was another concern. There is a substantial educational component to training a large number of physicians/NPPs on how to use this system. Proceduralists are not used to billing time-based codes nor, because of the global periods, are they used to billing for inpatient hospital visits other than as a consulting physician. Also, the new codes will have to be available within the various EMR and billing software that physicians/NPPs typically use.

\section{Next Steps}

To better understand the complexity of the coding task, it would be useful to pilot test the proposed codes in this report with individual proceduralists and practices. One approach would be to create a series of vignettes that represent the range of care that can be provided at postoperative visits. This testing would also allow us to assess interrater reliability (i.e., whether different physicians/NPPs apply the same code to the same vignette). In addition, such testing could help inform refinements of the codes to make them easier to use and, therefore, address concerns about underuse. The codes are proposed for the purpose of data collection and to inform the assessment of the adequacy of surgical global payments. They were not proposed to be used for payment to individual physicians/NPPs. If in the future they were used for payment purposes, the codes would require further testing and refinement.

\section{Conclusion}

Based on input from interviews with proceduralists and other physicians/NPPs who contribute to post-operative care, as well as an expert panel, we recommend a set of eight codes that CMS can use to collect data on post-operative care. The structure of these codes is based on a hybrid approach of using time, scope of care, and setting. The codes include three inpatient codes, three office-based visit codes, and two codes for care provided by telephone or electronically. 


\section{References}

AMA—see American Medical Association.

American Medical Association, "The RVS Update Process Committee," web page, updated 2016. As of May 5, 2016:

http://www.ama-assn.org/ama/pub/physician-resources/solutions-managing-yourpractice/coding-billing-insurance/medicare/the-resource-based-relative-value-scale/the-rvsupdate-committee.page

Berenson R. A., P. Basch, and A. Sussex, "Revisiting E\&M Visit Guidelines-A Missing Piece of Payment Reform," New England Journal of Medicine, 2011, Vol. 364, No. 20, pp. 18921895.

Berenson, R. A., and J. D. Goodson, "Finding Value in Unexpected Places-Fixing the Medicare Physician Fee Schedule," New England Journal of Medicine, Vol. 374, No. 14, 2016, pp. 1306-1309.

Brett, A.S., "New Guidelines for Coding Physicians' Services-A Step Backward," New England Journal of Medicine, Vol. 339, No. 23, 1998, pp. 1705-1708.

Centers for Disease Control and Prevention, “Ambulatory Health Care Data," web page, updated September 23, 2015. As of May 5, 2016:

http://www.cdc.gov/nchs/ahcd.htm

Centers for Medicare \& Medicaid Services, "Chapter 12-Physicians/Nonphysician

Practitioners," Medicare Claims Processing Manual, Baltimore, Md.: Center for Medicare and Medicaid Services, 2015, pp. 9-225. As of April 25, 2014:

http://www.cms.gov/Regulations-and-

Guidance/Guidance/Manuals/Downloads/clm104c12.pdf

_, CMS Manual System: Pub 100-04 Medicare Claims Processing, Transmittal 2395

(replaces Transmittal 1316), Baltimore, Md., August 27, 2007. As of May 5, 2014:

http://www.cms.gov/Regulations-and-

Guidance/Guidance/Transmittals/downloads/R2395CP.pdf

CMS—see Centers for Medicare \& Medicaid Services.

Code of Federal Regulations, Title 42, Centers for Medicare \& Medicaid Services, Parts 403, 405, 410, et al., Medicare Program, Revisions to Payment Policies Under the Physician Fee Schedule, Clinical Laboratory Fee Schedule, Access to Identifiables Data for the Center for Medicare and Medicaid Innovation Models and Other Revisions to Part B for CY 2015, Final 
Rule, Vol. 79, No. 219. November 2014, pp. 67547-68092. As of May 25, 2016 :

https://www.gpo.gov/fdsys/pkg/FR-2014-11-13/pdf/2014-26183.pdf

Cromwell, J., N. McCall, K. Dalton, and P. Braun, "Missing Productivity Gains in the Medicare Physician Fee Schedule: Where Are They?" Medical Care Research Review, Vol. 67, No. 6, 2010, pp. 676-693.

Crotty, B. H., Y. Tamrat, A. Mostaghimi, C. Safran, and B. E. Landon, "Patient-to-Physician Messaging: Volume Nearly Tripled as More Patients Joined System, but Per Capita Rate Plateaued," Health Affairs, Vol. 33, No. 10, 2014, pp. 1817-1822.

Gilchrist, V. J., K. C. Stange, S. A. Flocke, G. McCord, and C. C. Bourguet, “A Comparison of the National Ambulatory Medical Care Survey (NAMCS) Measurement Approach with Direct Observation of Outpatient Visits," Medical Care, 2004, pp. 276-280.

Gooch, R. A. and J. M. Kahn, "ICU Bed Supply, Utilization, and Health Care Spending: An Example of Demand Elasticity," Journal of the American Medical Association, Vol. 311, No. 6, 2014, pp. 567-568.

Gottschalk, A., and S. A. Flocke, "Time Spent in Face-to-Face Patient Care and Work Outside the Examination Room," Annals of Family Medicine, 2005, pp. 488-493.

Mafi, J. N., E. P. McCarthy, R. B. Davis, and B. E. Landon, "Worsening Trends in the Management and Treatment of Back Pain," JAMA Internal Medicine, September 23, 2013, pp. 1573-1581.

Mafi, J. N., S. T. Edwards, N. P. Pedersen, R. B. Davis, E. P. McCarthy, and B. E. Landon, "Trends in the Ambulatory Management of Headache: Analysis of NAMCS and NHAMCS Data 1999-2010," Journal of General Internal Medicine, January 8, 2015, pp. 1-8. As of May 5, 2016:

http://link.springer.com/10.1007/s11606-014-3107-3

Mafi, J. N., C. Wee, R. B. Davis, and B. E. Landon, "Comparing Use of Low Value Health Services Among U.S. Advanced Practice Clinicians and Physicians," Annals of Internal Medicine, forthcoming.

Medicare Learning Network, "Global Surgery Fact Sheet," Centers for Medicare \& Medicaid Services, No. ICN 907166, March 2015. As of May 16, 2016 :

https://www.cms.gov/Outreach-and-Education/Medicare-Learning-NetworkMLN/MLNProducts/downloads/GloballSurgery-ICN907166.pdf

Morrow, M., and S. Smith, American Medical Association, "Re: Methods Used to Summarize Post-Operative E/M Visits by CPT Code," email to Ashley Kranz, RAND Corporation, Arlington, Va., April 25, 2016. 
Office of the Inspector General, Cardiovascular Global Surgery Fees Often Did Not Reflect the Number of Evaluation and Management Services Provided, Report A-05-09-00054, U.S. Department of Health and Human Services, Washington, D.C., May 2012a. As of May 5, 2016:

http://oig.hhs.gov/oas/reports/region5/50900054.pdf

—_, Musculoskeletal Global Surgery Fees Often Did Not Reflect the Number of Evaluation and Management Services Provided, Report Number A-05-09-00053, U.S. Department of Health and Human Services, Washington, D.C., May 2012b. As of May 5, 2016: http://oig.hhs.gov/oas/reports/region5/50900053.pdf

Public Law 114-10, Medicare Access and CHIP Reauthorization Act of 2015, April 16, 2015. https://www.congress.gov/114/plaws/publ10/PLAW-114publ10.pdf 